\title{
Ein Esel ist kein kleines Pferd - Konsequenzen für die tierärztliche Behandlung
}

\author{
Laura Listmann
}

Obwohl sich der Esel als Haustier in Deutschland immer größerer Beliebtheit erfreut, gilt er bei einigen Tierärzten weiterhin als Exot, der viel zu häufig als kleines Pferd behandelt und auch therapiert wird. Neben vielen Gemeinsamkeiten gibt es jedoch einige wesentliche Unterschiede zwischen Pferd und Esel, die nicht nur in einer teilweise abweichenden Anatomie, sondern auch im Verhalten, in der Physiologie sowie in der Pharmakokinetik bestehen.

\section{Unterschiede zwischen Esel, Pferd und Maultier}

Der Esel unterscheidet sich vom Pferd durch seine langen Ohren und seine besondere Färbung des Kopfes. Das Fell im Bereich von Augen und Maul ist heller, kürzer und sehr fein. Statt eines Schopfes besitzt der Esel nur eine kurze Stehmähne und einen einer Rute gleichenden Schwanz ( Abb. 1). Im Bereich der Flanke lässt sich beim Esel kein Wirbel im Fell finden und die Kastanien sind bei ihm lediglich an den Vordergliedmaßen entwickelt. Im Gegensatz zum Pferd hat der Esel eine steilere Schulter und einen kürzeren kräftigen
Hals sowie einen flacheren Widerrist. Zudem besitzen männliche Tiere $\mathbf{2}$ Zitzen am Präputium, die nicht mit einem equinen Sarkoid verwechselt werden dürfen. Die Hufe der Esel sind kleiner und durch eine dickere Hufwand und einem steileren Hufwinkel gekennzeichnet [3].

Der Esel wird im Gegensatz zum Pferd deutlich älter und kann bei guter Haltung durchaus ein Alter von $\mathbf{4 5}$ Jahren und mehr erreichen.

Das Maultier entsteht aus einer Kreuzung von einem Eselhengst und einer Pferdestute und vereinigt Merkmale beider Spezies in unterschiedlicher Ausprägung ( Abb. 2). Als Maulesel wird dagegen die Kreuzung einer Eselstute mit einem Pferdehengst bezeichnet. Maultier und Maulesel gelten aus biologischer Sicht als Hybride und sind bis auf seltene Ausnahmen nicht fortpflanzungsfähig.

Auch genetisch unterscheiden sich Pferd, Esel und Maultier: Während das Pferd 64 Chromosomenpaare besitzt, hat der Esel lediglich 62, das Maultier hingegen 63.

\section{Anatomische Besonderheiten}

Der Tränennasenkanal endet beim Esel eher lateral und leicht dorsal im Nasenloch.

Der Winkel zwischen Pharynx und Larynx ist beim Esel mit $5,2^{\circ}$ steiler als beim Pferd mit $2,5^{\circ}$. Zusätzlich existiert ein Rec. pharyngeus zentral im Pharynx zwischen den Öffnungen der Luftsäcke. Diese Besonderheiten erschweren die Anwendung der Nasenschlundsonde, da man leicht in den Rec. pharyngeus gelangt, was die weitere Passage zum Oesophagus verhindert [10].

Der Esel besitzt einen stark ausgebildeten M. cutaneus colli, der das mittlere Drittel der Jugularvene verdeckt. Daher ist es beim Esel besonders wichtig, das obere Drittel der Jugularvene zur Venenpunktion zu verwenden und im Vergleich zum Pferd die Kanüle in einem steileren Winkel anzusetzen [2].

Beim Esel ist der erste Schwanzwirbel oft mit dem Kreuzbein fusioniert. Eine Epiduralanästhesie sollte also nicht wie beim Pferd im 1., sondern im 2. Intercoccygeal-

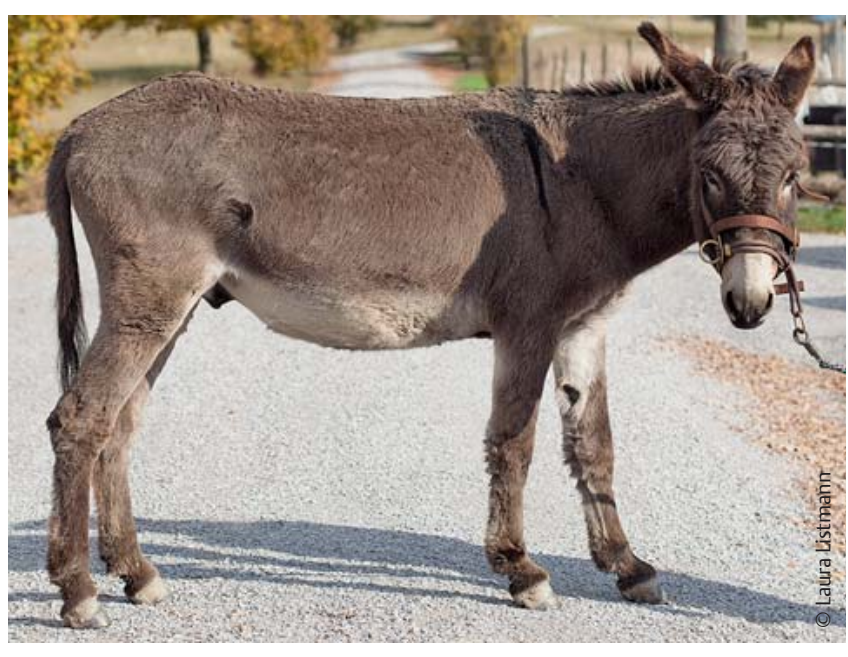

Abb. 1 Esel mit der typischen kurzen Stehmähne und dem einer Rute ähnelnden Schwanz.

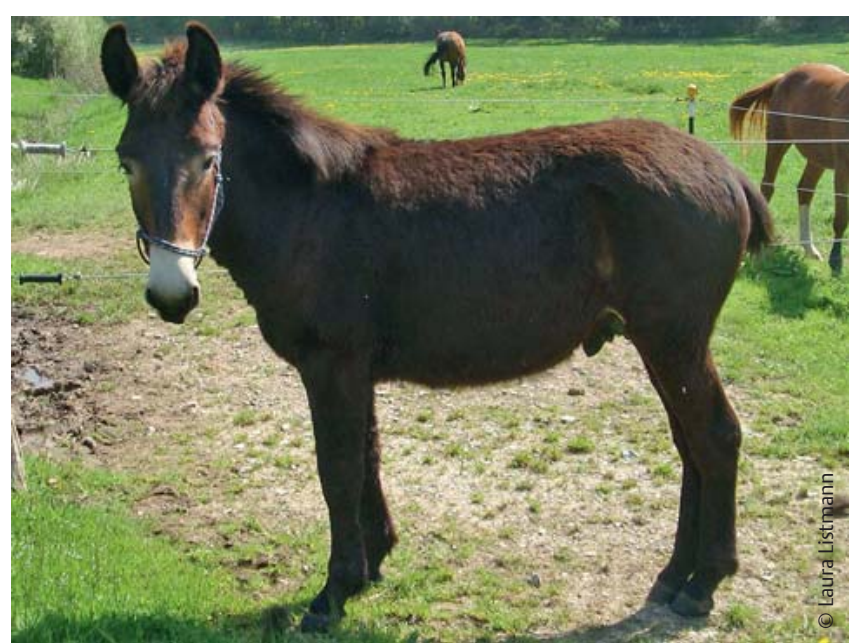

Abb. 2 Maultiere entstehen aus einer Kreuzung aus Eselhengst und Pferdestute, hier ein junger Maultierhengst. 
raum zwischen $\mathbf{C} 1$ und $\mathbf{C} 2$ erfolgen. Der Einstich erfolgt hier mit ca. $30^{\circ}$ etwas flacher [15].

Die Eselhoden sind im Vergleich zu denen des Pferdes größer und weisen eine sehr ausgeprägte Blutversorgung und damit eine erhöhte Blutungsneigung auf. Bei einer Kastration muss deshalb in jedem Fall eine Ligatur der Samenstranggefäße erfolgen, da es ansonsten in der Folge zum Verbluten des Esels kommen kann. Esel werden häufig nach einer Kastration im Stehen mit Samenstrangfisteln vorstellig, da der Samenstrang nicht korrekt abgesetzt wurde ( $\bullet$ Abb.4). Eine Kastration sollte deshalb im Liegen unter Allgemeinanästhesie durchgeführt werden, um ein sorgfältiges Ligieren der Samenstranggefäße und ein möglichst kurzes Absetzen des Samenstrangs zu gewährleisten $(\triangleright$ Abb. 5).

\section{Physiologische Besonderheiten}

Esel und Pferd unterscheiden sich auch in einigen physiologischen Eigenschaften [11].

Die Körperinnentemperatur des Esels unterliegt tageszyklischen Schwankungen und kann sich je nach Außentemperatur und Wasserhaushalt anpassen. Als physiologisch gelten Werte zwischen 36 und $40^{\circ} \mathrm{C}$ mit einem Mittelwert von $37^{\circ} \mathrm{C}$ [2]. Außerdem kann der Esel sein Plasmavolumen auch im Falle einer Dehydratation von $20 \%$ des Körperwassers stabil halten, während Pferde auf einen solchen Verlust sehr empfindlich reagieren [11]. Somit ist der Esel sehr gut an warme Klimazonen mit geringem Wasservorkommen angepasst.

Die Blutwerte des Esels weichen teilweise von denen des Pferdes ab. Referenzwerte stellt z.B. die Donkey Sanctuary in England zur Verfügung (www.thedonkeysanctuary.org.uk).

\section{Verhaltenstypische Merkmale}

Obwohl der Esel wie das Pferd ein Beutetier ist, verhält er sich in Gefahrensituationen häufig kontrovers. Anstatt vor der potenziellen Gefahr zu fliehen, bleibt er oft
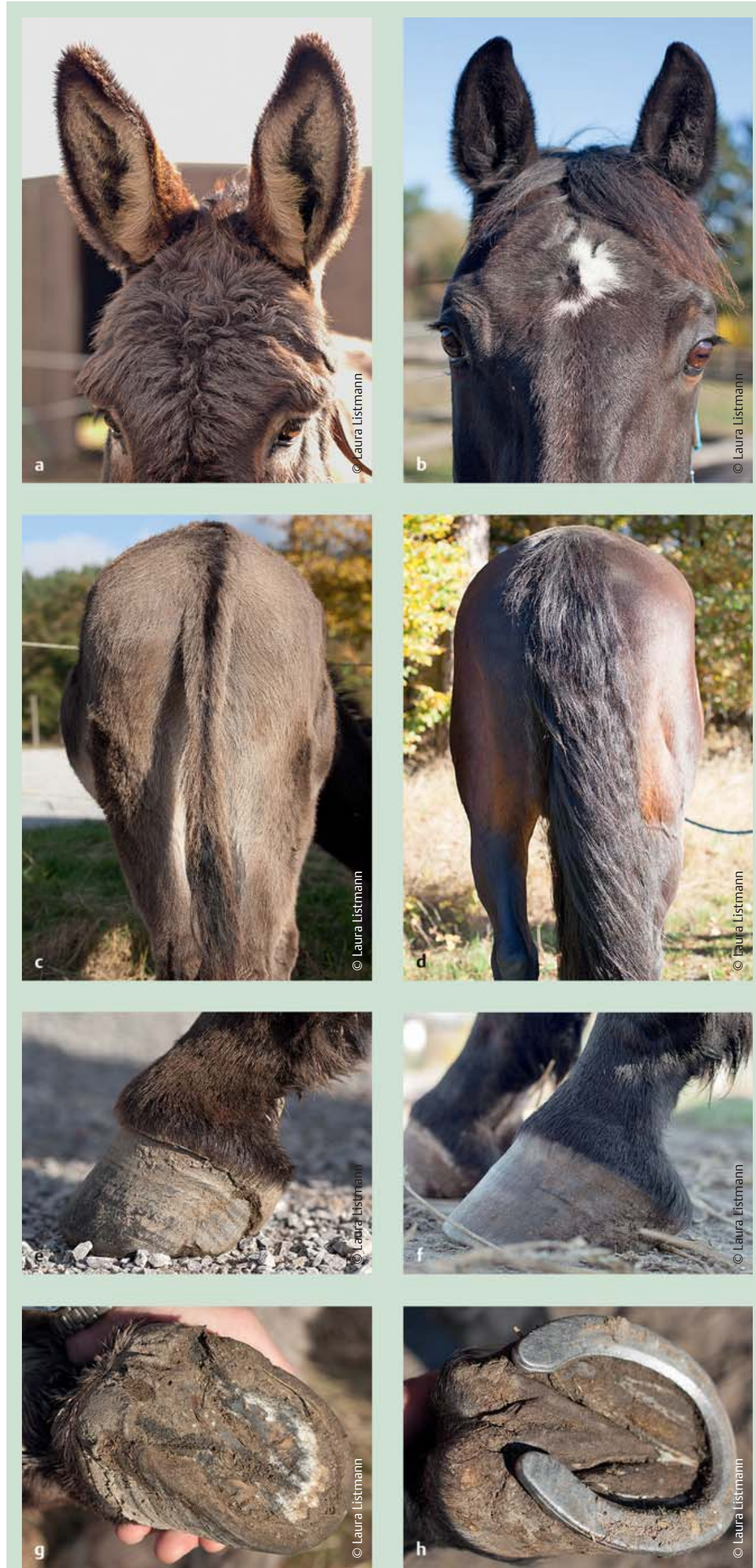

Abb. $\mathbf{a}$ a bis $\mathbf{h}$ Esel und Pferd im Vergleich. 


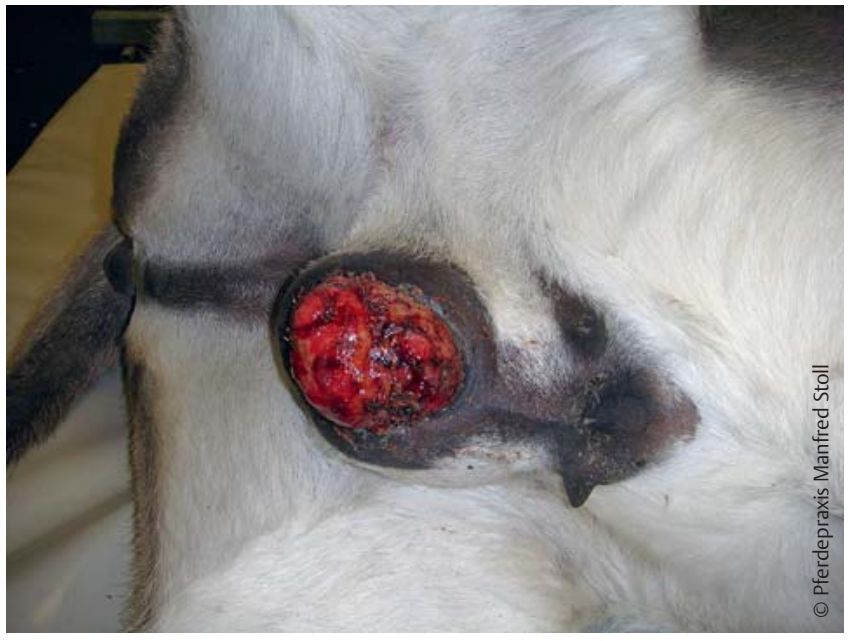

Abb. 4 Samenstrangfistel nach einer Kastration im Stehen.

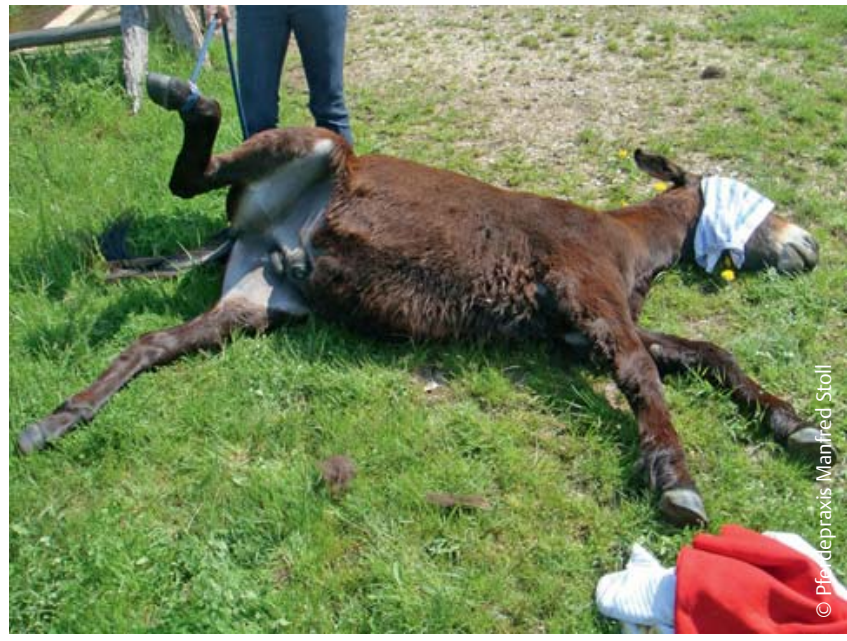

Abb. 5 Esel sollten im Liegen unter Allgemeinanästhesie kastriert werden, hier ein Maultier auf der Weide. wie angewurzelt stehen und kann sogar durch Treten mit den Vorderhufen und Beißen zum Angriff übergehen. Dieses Verhalten wird immer wieder mit Sturheit verwechselt.

Esel leben in lockeren Gruppen von bis zu 10 Tieren zusammen, wobei es innerhalb der Gruppe zu festen langjährigen Partnerschaften zwischen 2 Tieren kommen kann. Eselstuten und Wallache können deshalb gut in kleinen Gruppen gehalten werden. Eine Einzelhaltung oder Haltung mit artfremden Tieren führt zu einem fehlerhaften Sozialverhalten und sollte daher vermieden werden.

$\mathrm{Ab}$ dem Eintritt in die Geschlechtsreife, mit ca. 10-12 Monaten, weisen Eselhengste ein deutlich höheres Territorialverhalten auf und verhalten sich im Vergleich zu Pferdehengsten aggressiver gegenüber anderen Tieren und dem Menschen.

Die Haltung und der Umgang mit Eselhengsten gehört nur in die Hand von erfahrenen Personen.

Darüber hinaus versteht sich der Esel sehr gut darin, seine Schmerzen zu verbergen. Das geringe Repertoire an klinischen Symptomen kann den Tierarzt bei der Diagnosestellung vor große Herausforderungen stellen [14]. Schwerwiegende Erkrankungen, wie Koliken oder schwere Hufrehen, werden oftmals zu spät oder gar nicht erkannt. Auch scheinbar harmlose Symptome, wie vermindertes Allge- meinbefinden und Appetitlosigkeit, müssen infolgedessen sehr ernst genommen werden, da sie bereits einen Notfall darstellen können.

\section{Erkrankungen und deren klinische Symptome}

\section{Zahnprobleme}

Die weitaus häufigsten Erkrankungen beim Esel sind Zahnerkrankungen [14]. Dabei kommt es häufig vor, dass sogar schwerwiegende Zahnerkrankungen nicht erkannt werden, da der Esel keine Veränderungen bei der Nahrungsaufnahme zeigt. Tatsächlich ist es aber so, dass Esel die Futteraufnahme erst im hochgradigen Stadium bei unerträglichen Schmerzen einstellen. Eine regelmäßige sorgfältige Zahnkontrolle ist somit auch beim scheinbar gesunden Esel unerlässlich.

Der Esel unterscheidet sich in der Zahnanatomie und -histologie nicht wesentlich vom Pferd. Die Zähne des Esels sind hypsodont mit einem apikalen und einem koronalen Bereich, wobei der größte Teil der Zahnkrone als Reservekrone in der Alveole liegt. Der Esel hat 36-44 permanente Zähne, abhängig von der Ausbildung der Wolfszähne oder Canini. Zur Nummerierung und Identifikation der Zähne hat sich beim Esel das modifizierte Triadan-System nach Floyd bewährt [7].

\section{Der Oberkiefer ist breiter als der Unterkie-} fer, wodurch ein anisognathes Gebiss ent- steht, bei dem die Oberkieferbackenzähne die Unterkieferbackenzähne nach bukkal überragen [5]. Die 6 Backenzähne eines Kiefers bilden eine lückenlose Reihe und funktionieren als Kaublock. Um diesen lückenlosen Schluss zu gewährleisten, sind der erste und letzte Backenzahn leicht nach kaudal bzw. mesial verkippt und pressen gegen die Zahnreihe.

Wie beim Pferd beherbergen alle Schneidezähne je ein Infundibulum und die Backenzähne des Oberkiefers je 2 Infundibula. Auch die Position und die Anzahl der Pulpenkanäle gleichen denen des Pferdes.

\section{Erkrankungen der Schneidezähne}

$\mathrm{Zu}$ den Erkrankungen im Bereich der Schneidezähne zählen [5]:

- Fehlstellungen wie Brachy- und Prognathismus

- überlange Zähne

- fehlerhafte Abnutzung in Form eines Diagonalbisses

- Frakturen im Bereich der Schneidezähne und des Os incisivum bzw. der Mandibula

- überzählige oder fehlende Schneidezähne

- persistierende Milchschneidezähne

- dorsale oder ventrale Biegung der Schneidezahnlinie (,Frown` bzw. ,Smile')

- Ein leichter ,Smile‘ an den Schneidezähnen wird beim Esel im Gegensatz zum Pferd als physiologisch angesehen und sollte nicht korrigiert werden. 


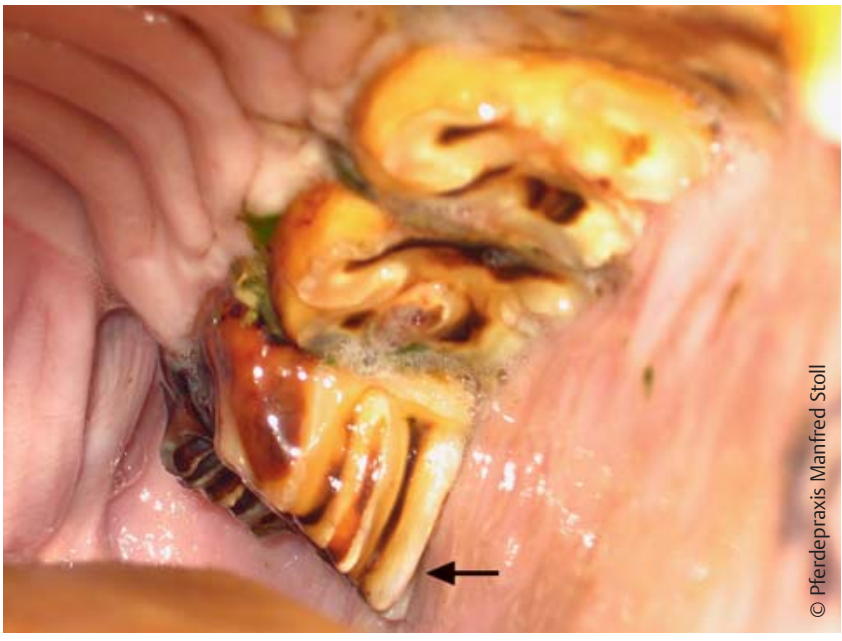

Abb. 6 Esel mit einem Meißelzahn $(\leftarrow)$ an Backenzahn 110.

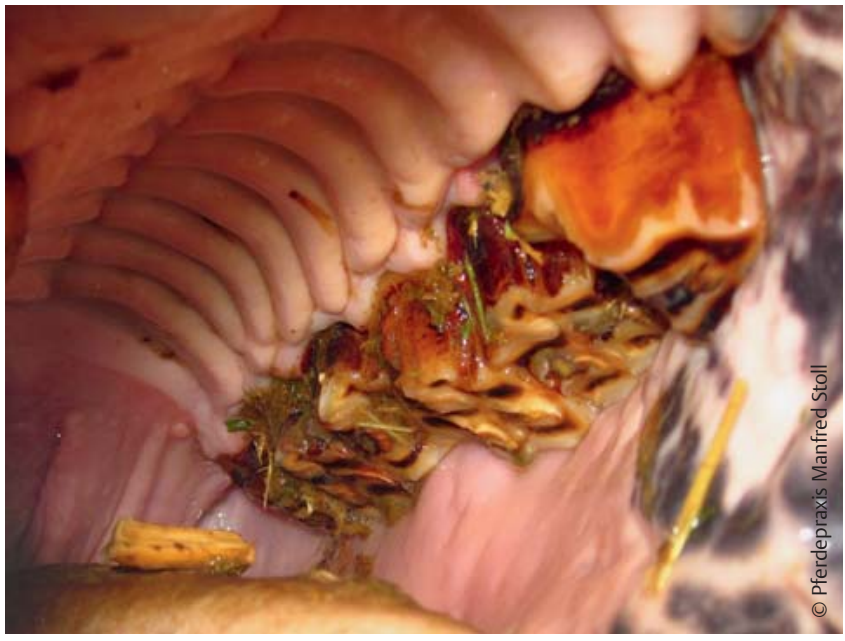

Abb. 7 Massives Wellengebiss mit Parodontaltaschen bei einem Esel.

\section{Erkrankungen der Backenzähne}

Im Bereich der Backenzähne sind die häufigsten Ursachen für Zahnprobleme:

- scharfe Zahnkanten ( Abb. 6)

- fehlerhafte oder mangelnde Abnutzung der Backenzähne mit Bildung eines Stufen- oder Wellengebisses ( A Abb. 7)

- pathologische Veränderungen des bukkolingualen Zahnwinkels

Dies kann zu schmerzhaften Verletzungen der Maulschleimhaut und der Zunge und zu Problemen beim Kauvorgang führen. Die Backenzähne sollten regelmäßig kontrolliert und ggf. durch Einschleiftherapie mit manuellen oder elektronischen Zahnraspeln korrigiert werden.

Sehr schmerzhaft sind Parodontaltaschen im Bereich der Backenzähne mit oder ohne Futtereinspießung. Sie entstehen durch die Lockerung, das Verkippen, das Verdrehen oder die Wanderung benachbarter Backenzähne und können im schlimmsten Fall zum Verlust betroffener Zähne führen. Die Therapie gleicht der beim Pferd [5].

Weitere Erkrankungen im Backenzahnbereich sind:

- Frakturen

- Luxationen

- apikale Infektionen mit und ohne Eröffnung der Pulpenposition

- infundibuläre oder periphere Karies

- dental bedingte Sinusitiden durch Zahnwurzelentzündungen
Auch hier gleicht die Therapie der des Pferdes.

\section{Kolik}

Die klinischen Symptome einer Kolik beim Esel unterscheiden sich oftmals stark von denen des Pferdes. Die Symptome beschränken sich meist auf Appetitlosigkeit, reduziertes Allgemeinbefinden und vermehrtes Liegen. Starkes Schwitzen, Wälzen und Unruhe kommen eher selten vor.

Die Inzidenzrate von Koliken bei Eseln liegt bei 5,9\% im Jahr und ähnelt der bei Pferden. Die Mortalitätsrate hingegen liegt mit 51,1\% deutlich höher als beim Pferd (6,7-15,6\%).

Die häufigste Kolikursache beim Esel ist die Dickdarmobstipation. Auch hier liegt die Mortalitätsrate mit 51,4\% deutlich höher als beim Pferd [4]. Der Darmabschnitt mit den häufigsten Obstipationen ist die Beckenflexur (39,6\%), danach folgen Zäkum und großes und kleines Colon. Auch häufig beschrieben werden Torsionen und Strangulationen des Darms, etwas seltener Kolitis, Neoplasien des Darms und 'Grass Sickness' [14].

Eine mögliche Ursache für die hohe Mortalitätsrate kann der Mangel an eindeutigen klinischen Symptomen und damit eine falsche oder zu späte Diagnose sein. Als weitere Komplikation kommt insbesondere beim übergewichtigen Esel häufig eine sekundäre Hyperlipämie infolge der Futterverweigerung hinzu. Eine Blutunter- suchung mit Triglyceridbestimmung ist deshalb sinnvoll.

Vorsicht ist bei der Interpretation des Hämatokritwerts geboten. Da der Esel sein Plasmavolumen bei mildem bis moderatem Flüssigkeitsverlust stabil hält, kann auch bei normalem Hämatokrit bereits eine Dehydratation vorliegen [12].

Ein direkter Zusammenhang konnte zwischen Dickdarmobstipation und Zahnproblemen bzw. zwischen gastrointestinalen Entzündungen und einem Befall mit Strongyliden festgestellt werden [14].

Nach einer überstandenen Kolik sollten auf jeden Fall eine sorgfältige Zahnkontrolle und Kotuntersuchung erfolgen.

\section{Lahmheiten/Hufrehe}

Lahmheiten und Hufprobleme stehen beim Esel an 4. Stelle der häufigsten Erkrankungen [14]. Hierbei sind Hufrehe und Hufabszesse in unseren Breitengraden die häufigsten Lahmheitsursachen.

\section{Hufrehe}

Die Diagnose einer Hufrehe beim Esel insbesondere bei beidseitiger Lahmheit ist häufig sehr schwierig, da sich die Symptome auf einen steifen Gang, stures Stehen an einer Stelle oder vermehrtes Liegen beschränken. Deshalb wird eine akute Hufrehe oft mit Kreuzverschlag oder Kolik verwechselt. 
Die Ergebnisse der klinischen Untersuchung sind ähnlich wie beim Pferd:

- verstärkte Pulsation der Mittelfußarterie

vermehrte Wärme des Hufes

positive Zangenprobe.

Eine Hufbeinsenkung ist beim Esel im Gegensatz zum Pferd nur schwer am Kronrand zu palpieren. In chronischen Fällen kommt es zur Verformung der Hufkapsel und zu einer Ringbildung im Bereich des Hufhorns.

Röntgenologisch weicht der Eselhuf von dem des Pferdes wie folgt ab [3]:

1. Vorderhuf: der dorsale Hufwandwinkel ist mit $61,61^{\circ}$ und der dorsale Hufbeinwinkel mit $64,11^{\circ}$ steiler als beim Pferd.

2. Der Abstand zwischen Hufwand und Dorsalwand des Hufbeins beträgt ca. $16 \mathrm{~mm}$ und zwischen Kronsaum und Proc. extensorius ca. $10 \mathrm{~mm}$.

3. Hufwand und dorsale Hufbeinwand sind wie beim Pferd parallel.

$\mathrm{Zu}$ den pathologischen Veränderungen des Hufes infolge einer Hufrehe zählen wie beim Pferd eine Senkung und/oder Rotation des Hufbeins, eine „Krempenbildung“ an der Hufbeinspitze und eine Abflachung des dorsalen Hufwandwinkels [3].

Die Ätiologie der Hufrehe ist ähnlich der des Pferdes. Übergewicht und metabolische Erkrankungen, wie Insulinresistenz in Verbindung mit dem Equinen Metabolischen Syndrom (EMS) und dem Equinen Cushing Syndrom (ECS), sind die Hauptursachen. Esel erkranken aufgrund einer geringeren Insulinsensitivität jedoch noch leichter an EMS als Pferde. Die Diagnose von EMS und ECS erfolgt wie beim Pferd.

Auch die Therapie der Hufrehe, bestehend aus Schmerztherapie, Hufverbänden, Hufbehandlung und geeigneter Fütterung, gleicht der des Pferdes. Bei der Verabreichung von NSAIDs ist der erhöhte Metabolismus zu beachten und die Wirkung daher regelmäßig zu überprüfen.

Esel erkranken aufgrund einer geringeren Insulinsensitivität leichter an EMS als Pferde.

\section{Hufabszesse}

Ursache, Diagnose und Behandlung von Hufabszessen sind wie beim Pferd. Hufabszesse entstehen beim Esel häufig durch subakute oder nicht erkannte chronische Hufrehen. Außerdem reagiert der Eselhuf besonders empfindlich auf Nässe, wie z.B. bei dauerhaft matschigem Boden. Auch mangelnde Hufpflege und Hufbehandlung sind beim Esel ein sehr häufiger Grund für Hufabszesse.

\section{Hyperlipämie}

Esel sind ähnlich wie Ponies sehr anfällig für Hyperlipämie. Zu den Risikofaktoren gehören, neben der niedrigeren Insulinsensitivität, Übergewicht, Stress bei Transport und Stallwechsel, Schmerzen, Hochträchtigkeit, Frühlaktation und weitere Erkrankungen wie Kolik, Zahnprobleme, Hufrehe und ECS. Da die Letalität bei $\mathbf{8 0} \%$ liegt, ist das Erkennen und Therapieren der Erkrankung im Frühstadium unerlässlich [1].

Die Symptome im Anfangsstadium umfassen meist nur Inappetenz und Mattigkeit, später folgen trockene verfärbte Schleimhäute, Halitosis, eine verminderte Peristaltik mit kleinen festen Kotballen, eine verminderte kapilläre Füllungszeit, Ödeme und schließlich Tachykardie, Tachypnoe und hepatische Enzephalopathie. Als Komplikation kommt es sekundär häufig zu Pankreatitis, Fettleber und Magengeschwüren.

Die Diagnose wird entweder durch die visuelle Untersuchung des trüb-milchig verfärbten Serums oder durch eine Triglyzeriderhöhung (>4,4 mmol/l) im Blut gestellt.

Die Therapie erfolgt im ersten Schritt durch Ausschalten der Krankheitsursache. Je nach Schweregrad der Hyperlipämie folgen:

- Infusionen i.v. oder per Nasenschlundsonde

- Verabreichung von Glukose (100 g Glucose $+5 \mathrm{~g}$ Natriumbicarbonat $+12,5 \mathrm{ml}$ Vitaminkonzentrat $+2-31$ Wasser per Nasenschlundsonde $2 \times$ tgl.)

- Flunixin-Meglumin in anti-endotoxischer Dosis: $0,25 \mathrm{mg} / \mathrm{kg}$ i.v. $2 \times \mathrm{tgl}$.

- Insulin: 0,1-0,3 IU/kg s.c.

- Heparin: 100-200 IU/kg i.v. und

- Omeprazol: $4 \mathrm{mg} / \mathrm{kg}$ p.o. [8].

\section{Atemwegserkrankungen}

Die infektiösen bakteriellen und viralen Atemwegserkrankungen beim Esel gleichen in Vorkommen, Diagnose und Therapie denen des Pferdes.

Wie das Pferd kann auch der Esel an chronisch obstruktiver Bronchitis (COB) erkranken. Die Krankheitsursache, der Verlauf und die Therapie sind wie beim Pferd. Da Esel weniger für sportliche Aktivitäten genutzt werden, bleiben Erkrankungen des Respirationstrakts häufig lange unentdeckt und werden erst im chronischen Stadium erkannt.

Einen Unterschied zum Pferd macht die insbesondere beim älteren Esel vorkommende idiopathische Lungenfibrose mit einer Prävalenz von 35\% [13]. Diese betrifft die Subpleura und das interstitielle Lungengewebe. Die Ursachen sind weitgehend ungeklärt, jedoch wird das asine Herpesvirus $\mathbf{4}$ und $\mathbf{5}$ mit der Lungenfibrose in Verbindung gebracht [9]. Die klinischen Symptome sind ähnlich der COB. Eine Therapie gibt es nicht. Eine medikamentelle Behandlung wie bei der COB und bestmögliche Haltungsbedingungen können die Symptome jedoch etwas lindern.

\section{Pharmakokinetik}

Esel scheinen einen erhöhten Metabolismus für verschiedene Medikamente $\mathrm{zu}$ besitzen, der auf einen Unterschied im Cytochrom-P450-Isoenzym zurückzuführen ist. Dosis und Intervall einiger Medikationen müssen daher entsprechend angepasst werden. Obwohl es mittlerweile mehrere Studien zur Dosierung bestimmter Medikamente beim Esel gibt, beruht das Dosierungsmanagement häufig noch immer auf Schätzungen oder persönlichen Erfahrungen [11].

\section{Antibiotika}

Wie beim Pferd sollten Antibiotika auch beim Esel gemäß den Antibiotika-Leitlinien nach Antibiogramm eingesetzt werden. Die gängigsten Antibiotika gibt - Tab. 1 wieder.

\section{NSAIDs}

Einige NSAIDs werden beim Esel schneller metabolisiert und müssen deshalb bei unzureichender Analgesie häufiger verab- 
Tab. 1 Dosierungsvorschläge für Antibiotika beim Esel (Donkey Sanctuary, England).

\begin{tabular}{|c|c|}
\hline Wirkstoff & Dosierung \\
\hline Cefquinom & 1 mg/kg 1-2 × tgl. i.m. \\
\hline Enrofloxacin & 7,5 mg/kg $1 \times$ tgl. p. o./i.v. \\
\hline Gentamicin & 6,6 mg/kg $1 \times$ tgl. i. v. \\
\hline Oxytetracyclin & $\begin{array}{l}5-7 \text { mg/kg } 2 \times \text { tgl. } \\
\text { langsam i. v. }\end{array}$ \\
\hline $\begin{array}{l}\text { Procain- } \\
\text { Penicillin G }\end{array}$ & $10-20 \mathrm{mg} / \mathrm{kg} 1 \times$ tgl. i. m. \\
\hline $\begin{array}{l}\text { Trimethoprim/ } \\
\text { Sulfonamid }\end{array}$ & 30 mg/kg 1-2 × tgl. p.o. \\
\hline
\end{tabular}

Tab. 2 Dosierungsvorschläge für NSAIDs beim Esel (Donkey Sanctuary, England).

\begin{tabular}{|c|c|}
\hline Wirkstoff & Dosierung \\
\hline Carprofen & $\begin{array}{l}0,7 \mathrm{mg} / \mathrm{kg} \text { einmalig i. v. oder } \\
1 \times \text { tgl. p. o. für 4-9 Tage }\end{array}$ \\
\hline $\begin{array}{l}\text { Flunixin- } \\
\text { Meglumin }\end{array}$ & $\begin{array}{l}1,1 \mathrm{mg} / \mathrm{kg} 1-2 \times \text { tgl. i.v./ } \\
\text { p.o. }\end{array}$ \\
\hline $\begin{array}{l}\text { Phenyl- } \\
\text { butazon }\end{array}$ & $\begin{array}{l}\text { 4,4 mg/kg initial p. o./i. v., } \\
\text { danach } 2,2 \mathrm{mg} / \mathrm{kg} 2-3 \times \text { tgl. } \\
\text { p. o. }\end{array}$ \\
\hline
\end{tabular}

reicht werden ( $\triangleright$ Tab.2). Eine genaue Kontrolle der Wirkung ist wichtig, um die Dosis ggf. anpassen zu können.

\section{Sedativa}

Für eine Sedation beim Esel können alle für das Pferd verfügbaren $\boldsymbol{\alpha} \mathbf{2}$-Agonisten eingesetzt werden.

\section{für kürzere Eingriffe:}

- Xylazin: 0,4-1,1 mg/kg i.v.

\section{für längere Eingriffe:}

- Detomidin: 0,02-0,04 mg/kg i.v. oder

- Romifidin: 0,04-0,12 mg/kg i.v. [12]

für eine tiefe Sedierung mit stärkerer Analgesie:

- Kombination mit Butorphanol: 0,02$0,04 \mathrm{mg} / \mathrm{kg}$ i.v. [6]

Um eine adäquate Sedierung zu erreichen, kann die Dosierung insbesondere beim Maultier bis zu 50\% höher sein als beim Pferd [11].

Wie beim Pferd sind eine ruhige Umgebung und sanftes Handling vor der Seda-

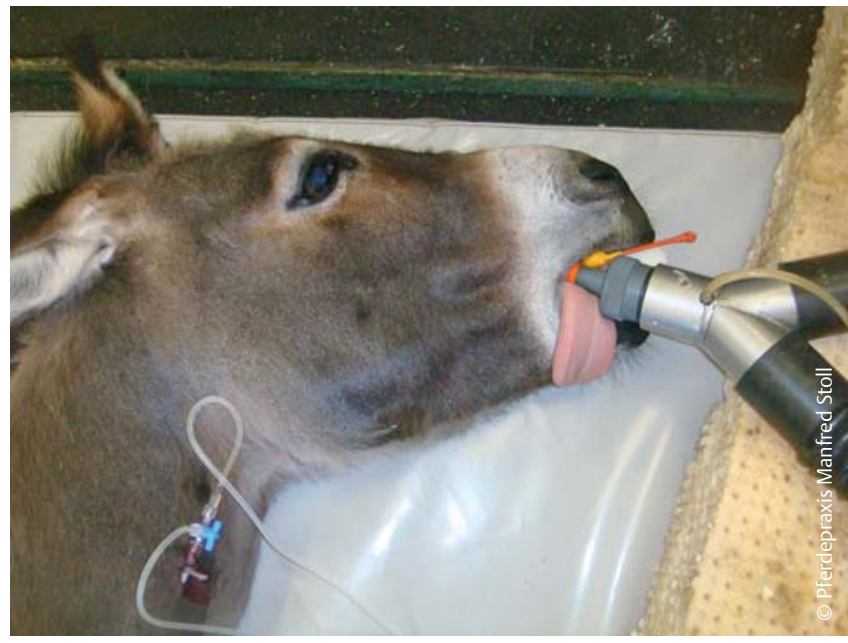

Abb. 8 Für die Intubation eines Esels wird aufgrund des engen Naseneingangs und des abweichenden Larynxwinkels ein eher kleiner Tubus verwendet (14$18 \mathrm{~mm}$ Innendurchmesser).

tion wichtig, um eine bestmögliche Wirkung zu erzielen. Stress vor der Sedierung und während der Injektion sollte unbedingt vermieden werden, da Esel darauf mit einer Hyperlipämie reagieren können.

Die Anwendung von Lokalanästhetika bei schmerzhaften Eingriffen ist sinnvoll, um die Dosis von Sedativa und Anästhetika zu senken. Sie gleichen in Dosis und Anwendung der des Pferdes. Da Esel durch ihr stoisches Verhalten wenig Schmerzsymptome zeigen, darf nach operativen Eingriffen eine Analgesie mit entsprechenden NSAIDs nicht vergessen werden.

\section{Anästhetika}

Für die Allgemeinanästhesie ist eine gründliche prä-operative Untersuchung unerlässlich, die ggf. durch eine Blutuntersuchung ergänzt werden sollte, da durch das bereits erwähnte stoische Verhalten des Esels der tatsächliche Krankheitszustand oft schwer zu beurteilen ist.

\section{Injektionsnarkosen}

Esel metabolisieren Ketamin schneller als Pferde, sind aber sensibler gegenüber Guaifenesin [12].

Generell kann gesagt werden, dass kleine Esel eine höhere Dosis Anästhetika pro kg Körpergewicht verstoffwechseln als große Esel.

Wie beim Pferd gibt es verschiedene Kombinationen und Techniken für die Injektionsanästhesie beim Esel. Die Donkey Sanctuary schlägt folgende Narkose-Protokolle vor: für kurze Eingriffe (bspw. Kastrationen auf der Weide):

- Prämedikation mit Xylazin oder Detomidin kombiniert mit Butorphanol $(0,02-0,04 \mathrm{mg} / \mathrm{kg})$

- nach Eintreten der Sedierung (3$5 \mathrm{~min}$.): Ketamin (2,2 mg/kg i.v.) kombiniert mit Diazepam $(0,02-0,1 \mathrm{mg} / \mathrm{kg}$ i.v.)

für längere Eingriffe (bis zu 2 h):

- Einleitung

- Narkoseerhaltung mittels Triple-Drip (11 5\% Guaifenesin + 20 ml Ketamin [100 mg/ml] + $25 \mathrm{ml}$ Xylazin [20 mg/ $\mathrm{ml}]$ )

Bei Guaifenesin muss beachtet werden, dass die toxische Dosis bei $150 \mathrm{mg} / \mathrm{kg}$ und die letale Dosis bei $\mathbf{3 0 0} \mathbf{~ m g} / \mathbf{k g}$ erreicht wird [12].

\section{Inhalationsnarkosen}

Auch beim Esel eignet sich die Inhalationsanästhesie zur Verminderung der Dosis von Injektionsanästhetika. Präparate und Anwendungen entsprechen denen beim Pferd.

Die Intubation des Esels gestaltet sich aufgrund des engen Naseneingangs und des abweichenden Larynxwinkels gelegentlich schwieriger als beim Pferd. Wichtig ist hierbei, dass der Esel ausreichend anästhesiert ist und eine Auswahl an kleineren Tuben (14-18 mm Innendurchmesser) zur Verfügung steht $(\triangleright \mathbf{A b b} . \mathbf{8})$. 


\section{Entwurmung}

Die Endoparasiten des Esels entsprechen im Wesentlichen denen des Pferdes, sodass sich beide Spezies bei gemeinsamer Haltung gegenseitig infizieren können. Entwurmungsregime und Dosierung der Endoparasitika können vom Pferd übernommen werden. So sollte mindestens $1 \times$ jährlich eine Behandlung gegen Bandwürmer (z.B. mit Praziquantel) durchgeführt werden. Für eine gezielte Endoparasitenbehandlung ist eine vorherige Kotuntersuchung sinnvoll.

Eine Besonderheit stellt beim Esel der Befall mit Lungenwürmern dar: Esel gelten als natürliche Wirte und der Lungenwurm vollzieht im Esel einen kompletten Lebenszyklus, ohne dabei klinische Symptome auszulösen. Der Esel kann das Pferd bei gemeinsamer Haltung mit dem Lungenwurm infizieren. Die Diagnose sollte mittels Kotuntersuchung gestellt werden, die anschließende Behandlung erfolgt mit Ivermectin oder Moxidectin.

\section{Impfung}

Wie Pferde sind auch Esel sehr empfänglich für Tetanussporen. Esel sollten deshalb in jedem Fall gegen Tetanus geimpft werden.

Eine Impfung gegen das equine Influenza-, Tollwut- oder Herpesvirus sollte je nach Haltung und Kontakt zu bestandsfremden Tieren abgewogen werden.

\section{Fazit}

Bei der tierärztlichen Betreuung von Eseln ist zu beachten, dass auch kleine Abweichungen vom normalen Allgemeinverhalten Symptome leichter oder auch schwerer Erkrankungen sein können und Esel sensibel auf übermäßige Therapie und grobes Handling mit Futterverweigerung reagieren können. Durch den erhöhten Metabolismus verschiedener Medikamente ist eine genaue Überprüfung der Wirkung und dementsprechende Anpassung der notwendigen Dosis unerlässlich.

\section{Literatur}

1 Burden FA, du Toit N, Hazell-Smith E et al. Hyperlipemia in a population of aged donkeys: Description, pervalence and potential riskfactors. J Vet Intern Med 2011; 25: 1420-1425

2 Burnham SL. Anatomical differences of the mule and donkey. AAEP Proceedings 2002; 48: 102109

3 Collins SN, Dyson SJ, Murray RC et al. Radiological anatomy of the donkey's foot: Objective characterisation of the normal and laminatic donkey foot. Equine Vet J 2011; 43: 478-486

4 Cox R, Proudman CJ, Tawford AF et al. Epidemiology of impaction colic in donkeys in the UK. Vet Res 2007; $3: 1$

5 Du Toit N, Dixon P. Common dental disorders in donkeys. Equine Vet Educ 2012; 24: 45-51

6 El-Kammar MH, Gad SB. Evaluation of the sedative, analgesic, clinicophysiological and haematological effects of intravenous detomidine, detomidine-butorphanol, romifidine and romifidine-butorphanol in standing donkeys. Equine Vet Educ 2014; 26: 202-207
7 Floyd MR. The modified Triadansystem: nomenclature for veterinary dentistry. J Vet Dent 1991; 8(4): 18-19

8 Grove V. Hyperlipaemia. In: Svendsen ED, Hrsg. The professional handbook of the donkey. Yatesbury: Whittet Books; 2008: 52-61

9 Kleiboeker SB, Schommer SK, Johnson PJ et al. Association of two newly recognized herpes viruses with interstitial pneumonia in donkeys. J Vet Diagn Invest 2002; 14: 273-280

10 Lindsay EF, Clayton HM. An anatomical and endoscopic study of the nasopharynx and larynx of the donkey. J Anat1986; 144: 123-132

11 Lizarraga I, Sumano H, Brumbaugh W. Pharmacological and pharmacokinetic differences between donkeys and horses. Equine Vet Educ 2004; 16: 102-112

12 Matthews N. Anaesthesia and sedation. In: Svendsen ED, Hrsg. The professional handbook of the donkey. Yatesbury: Whittet Books; 2008 288-296

13 Miele A, Dhaliwal K, du Toit $N$ et al. Chronic pleuropulmonary fibrosis and elastosis of aged donkeys. Chest 2014; 145: 1325-1332

14 Morrow LD, Smith KC, Piercy RJ et al. Retrospective analysis of post-mortem findings in 1444 aged donkeys. J CompPath 2011; 144: 145-156

15 Shoukry M, Saleh M, Fouad K. Epiduralanesthesia in donkeys. Vet Rec 1975; 97: 450-452

\section{Online}

http://dx.doi.org/10.1055/s-0042-109439

\section{Laura Listmann}

Pferdepraxis Manfred Stoll

Bleidenstadter Weg 7

65329 Hohenstein 